\title{
Ubiquilin-2 Efficiently Lowers Levels of Common Disease Proteins, including the ALS-linked Protein TDP-43
}

Jacqueline Welday, ${ }^{*}$ Julia Gerson, Stephanie Sandoval-Pistorius, Jordan Gregory, and Henry Paulson, MD, PhD $\dagger$ Department of Neurology, Biomedical Sciences Research Building, Ann Arbor, MI, 48109

*To whom correspondence should be addressed: weldayj@umich.edu

†henryp@med.umich.edu

Ubiquilin-2 (UBQLN2) is a homeostatic regulatory protein that functions primarily in the ubiquitin-proteasome system and autophagy to shuttle ubiquitinated proteins for degradation. When mutated, UBQLN2 directly leads to frontotemporal dementia (FTD) and amyotrophic lateral sclerosis (ALS), which are adult-onset neurodegenerative diseases characterized primarily by the presence of trans-active DNA binding protein-43 (TDP-43) inclusions in the cytoplasm. To investigate the ability of UBQLN2 to regulate FTD/ALS pathology, we first transfected HEK-293 cells to express TDP-43 in combination with UBQLN2 or with UBQLN2 knocked-down. The ability of UBQLN2 to regulate other common aggregation-prone disease proteins, including tau and alpha-synuclein, was also evaluated. Co-expressed UBQLN2 markedly lowered levels of wildtype overexpressed TDP-43, as well as other common disease proteins tau and alpha-synuclein. Conversely, a knockdown of UBQLN2 via siRNA significantly elevated levels of wildtype TDP43 and tau, and robustly elevated levels of $\alpha$-synuclein. However, mutations in TDP43 decreased the ability of UBQLN2 to lower TDP43 levels. To confirm that UBQLN2 directly interacts with disease proteins, UBQLN2 was immunoprecipitated from HEK-293 cells expressing both UBQLN2 and either tau, $\alpha$-synuclein or TDP43. UBQLN2 was shown to directly interact with both tau and TDP43, but further experiments are needed to clarify the relationship between UBQLN2 and $\alpha$-synuclein. To determine how UBQLN2 regulates endogenous levels of disease proteins in vivo, UBQLN2 transgenic and UBQLN2 KO mice were analyzed for levels of TDP43, tau and $\alpha$-synuclein via Western blot. While soluble levels of TDP43, tau and $\alpha$-synuclein were unchanged, UBQLN2 lowered levels of endogenous, insoluble disease proteins when overexpressed. Ongoing studies are needed to confirm the relationship between UBQLN2 KO mice and their endogenous proteins, but preliminary results suggest that there is no significant difference in either soluble or insoluble levels between WT and UBQLN2 KO mice. Given that mutated UBQLN2 has been shown to directly cause FTD/ALS, understanding its role in toxic protein regulation may ultimately help elucidate disease progression and onset. Further study will evaluate how disease mutations alter the function of UBQLN2 in regulating disease proteins. Our findings indicate the potential importance of UBQLN2 in regulating ALS/FTD pathology and highlight a new role for regulating levels of other common neurodegenerative disease protein such as tau and $\alpha$-synuclein. 